\title{
Besoins de formation continue d'éducateurs physiques et à la santé
}

\section{The Needs for Continuing Education for Physical and Health Educators \\ Necesidades de formación continua de educadores físicos y para la salud}

\section{Sylvain Turcotte, Johanne Grenier, Marie-Claude Rivard, Charlotte Beaudoin, Martin Roy et Robert Goyette}

Volume 14, numéro 2, 2011

L'éducation à la santé en milieu scolaire : un champ de pratique et de recherche en émergence

URI : https://id.erudit.org/iderudit/1008913ar

DOI : https://doi.org/10.7202/1008913ar

Aller au sommaire du numéro

Éditeur(s)

Faculté d'éducation, Université de Sherbrooke

ISSN

1911-8805 (numérique)

Découvrir la revue

Citer cet article

Turcotte, S., Grenier, J., Rivard, M.-C., Beaudoin, C., Roy, M. \& Goyette, R. (2011). Besoins de formation continue d'éducateurs physiques et à la santé. Nouveaux cahiers de la recherche en éducation, 14(2), 79-95.

https://doi.org/10.7202/1008913ar

\section{Résumé de l'article}

L'objectif de cette étude consiste à décrire les besoins de formation continue (FC) des enseignants en éducation physique et à la santé (ÉPS) en matière d'éducation à la santé (ÉS) à travers l'éclairage du référentiel québécois des compétences professionnelles (CP). Les données issues d'un questionnaire complété par 223 enseignants d'ÉPS au primaire $(\mathrm{F}=80 ; \mathrm{H}=143)$ révèlent des besoins de FC nombreux et variés. La conception d'outils pédagogiques et l'évaluation des élèves associées aux compétences professionnelles de l'acte d'enseigner constituent leurs plus grandes préoccupations. Les enseignants souhaitent une formation qui assurerait un accompagnement individualisé adapté à leurs besoins. La perspective d'une culture de collaboration visant à créer un environnement où le développement professionnel continu prend vie à l'intérieur de l'école est explorée. 


\title{
Besoins de formation continue d'éducateurs physiques et à la santé
}

\author{
Sylvain Turcotte \\ Université de Sherbrooke \\ Johanne Grenier \\ Université du Québec à Montréal \\ Marie-Claude Rivard \\ Université du Québec à Trois-Rivières \\ Charlotte Beaudoin \\ Université d'Ottawa \\ Martin Roy \\ Université de Sherbrooke \\ et Robert Goyette \\ Université de Sherbrooke
}

\begin{abstract}
Besoins de formation continue d'éducateurs physiques et à la santé
Résumé

L'objectif de cette étude consiste à décrire les besoins de formation continue (FC) des enseignants en éducation physique et à la santé (ÉPS) en matière d'éducation à la santé (ÉS) à travers l'éclairage du référentiel québécois des compétences professionnelles $(\mathrm{CP})$. Les données issues d'un questionnaire complété par 223 enseignants d'ÉPS au primaire $(\mathrm{F}=80 ; \mathrm{H}=143)$ révèlent des besoins de $\mathrm{FC}$ nombreux et variés. La conception d'outils pédagogiques et l'évaluation des élèves associées aux compétences professionnelles de l'acte d'enseigner constituent leurs plus grandes préoccupations. Les enseignants souhaitent une formation qui assurerait un accompagnement individualisé adapté à leurs besoins. La perspective d'une culture de collaboration visant à créer un environnement où le développement professionnel continu prend vie à l'intérieur de l'école est explorée.
\end{abstract}

Mots-clés : formation continue, éducation à la santé, compétences professionnelles, enseignants, éducation physique et à la santé 


\section{The Needs for Continuing Education for Physical and Health Educators}

\section{Abstract}

This study aims at describing the needs for continuous education (FC) for physical and health education (ÉPS) teachers in the field of Health Education (ÉS) and filtered through the Québec benchmark of Professional Competencies (CP). The data from a questionnaire filled in by 223 ÉPS teachers at the elementary level $(\mathrm{F}=80 ; \mathrm{M}=143)$ reveal numerous and assorted $\mathrm{FC}$ needs. The teachers' most important concerns include the development of pedagogical tools and student assessments linked to the professional competencies related to the act of teaching. Teachers are looking for training that provides individualized support adapted to their needs. The study explores the perspective of a collaborative culture that would create an environment in which continuous professional development is offered within the school environment.

Key words: continuous education, health education, professional competencies, teachers, physical and health education

\section{Necesidades de formación continua de educadores físicos y para la salud}

\section{Resumen}

El objetivo del presente estudio es describir las necesidades de formación continua (FC) de los docentes en educación física y para la salud (EPS) respecto a la educación para la salud (ES) a través del enfoque del referencial de competencias profesionales de Quebec (CP). Los datos procedentes de un cuestionario llenado por 223 docentes de EPS en la primaria $(\mathrm{F}=80 ; \mathrm{H}=143)$ muestran que las necesidades de FC son numerosas y variadas. La concepción de instrumentos pedagógicos y la evaluación de los alumnos, asociadas a las competencias profesionales del acto de enseñar, constituyen sus preocupaciones las más grandes. A los docentes les gustaría poder contar con una formación que proporcionara un acompañamiento individualizado adaptado a sus necesidades. Se explora la perspectiva de una cultura de colaboración para crear un entorno donde el desarrollo profesional continuo queda plasmado dentro de la escuela.

Palabras clave: formación continua, educación para la salud, competencias profesionales, docentes, educación física y para la salud

\section{Introduction}

Depuis l'avènement du renouveau pédagogique au Québec, l'éducation à la santé (ÉS) comme champ d'études tient une place originale parmi les missions assignées au système éducatif, tel qu'en témoigne le curriculum d'éducation physique et à la santé (ÉPS). Malgré cette volonté politique, les acteurs des milieux scolaires reconnaissent qu'un nouveau curriculum ne suffit pas pour promouvoir de nouvelles approches éducatives. Tel que discuté par Jourdan (2004), «Ces textes risquent de rester lettre morte s'ils ne s'accompagnent pas de stratégies visant à permettre une implication réelle de l'ensemble des acteurs de l'école» (p. 19). Ainsi, la formation continue (FC) des enseignants d'ÉPS constitue un enjeu capital, une priorité sur laquelle s'accordent bon nombre de chercheurs (Beaudoin, Trudel et Mathias, 2006; Fullan, 2003; Turcotte, Gaudreau et Otis, 2007).

Dans un effort pour identifier les compétences professionnelles (CP) requises de la part d'un enseignant, le ministère de l'Éducation du Québec (MEQ, 2001b) a établi un référentiel de 12 compétences que le présent article utilise afin de poser un regard sur les besoins de FC exprimés par des enseignants d'ÉPS. 


\section{Problématique}

L'étude soulève le problème des besoins de FC en ÉS des enseignants d'ÉPS, depuis la transformation des programmes de formation des élèves aux ordres d'enseignement primaire et secondaire (MELS, 2007; MEQ, 2001a, 2003). Cette transformation est caractérisée par une approche par compétences qui s'appuie sur le constructivisme, le socioconstructivisme et le cognitivisme afin de mobiliser l'apprenant dans son processus d'apprentissage (Jonnaert, 2002). Pour sa part, l'éducation physique a été entièrement revisitée et le volet ÉS prend ancrage au sein de la compétence disciplinaire Adopter un mode de vie sain et actif. Cette compétence met au défi les enseignants d'ÉPS, car elle nécessite un élargissement des contenus et des pratiques pédagogiques (Turcotte et al., 2007).

De plus, le renouveau pédagogique a contribué à élargir le champ des CP requises de la part des enseignants. En effet, la nouvelle mission de l'école québécoise, soit instruire, socialiser et qualifier, redéfinit le rôle et les tâches de l'enseignant qui œuvre au sein d'une société québécoise hétérogène et changeante tant aux plans scolaire, familial, social et démographique que professionnel. Les orientations ministérielles se traduisent également par l'établissement d'un référentiel de $12 \mathrm{CP}$ (MEQ, 2001b) organisé en quatre catégories: Fondements, Acte d'enseigner, Contexte scolaire et social et Identité professionnelle. Les CP de l'identité professionnelle mettent justement en évidence la nécessité du renouvellement des pratiques pédagogiques, de la prise en charge du développement professionnel et de l'engagement autonome dans un plan de FC. Pour plusieurs, la FC apparaît essentielle à la mise en œuvre du renouveau pédagogique dans les milieux de pratique (Armour et Yelling, 2007; Bechtel et O'Sullivan, 2007; Fullan, 2003). Le ministère de l’Éducation du Québec définit la FC par «l'ensemble des actions et des activités dans lesquelles les enseignantes et les enseignants en exercice s'engagent de façon individuelle et collective en vue de mettre à jour et d'enrichir leur pratique professionnelle» (MEQ, 1999, p. 11).

Les quelques rares études qui se sont intéressées à l'impact de nouveaux curricula en ÉPS et à l'ajout de l'ÉS ont mis en évidence les besoins de FC chez les enseignants d'ÉPS (Beaudoin et al., 2006; Cogérino, 1999; Johns, 2003; Tinning, 2004). Leurs conclusions énoncent que les enseignants d'ÉPS ont été laissés sans ressource ni formation, alors que le manque de soutien financier et d'installations ainsi que le temps insuffisant d'enseignement en ÉPS sont demeurés inchangés. L'originalité de la présente étude repose donc sur l'utilisation du référentiel québécois des $12 \mathrm{CP}$ pour analyser les besoins de FC spécifiques de l'ÉS, auprès d'enseignants d'ÉPS.

\section{Cadre conceptuel}

La démarche de cette étude s'appuie sur les conclusions de travaux scientifiques (Beaudoin, Rivard, Grenier et Caty, 2008; Deslandes, 2001 ; Hargreaves, 2005; St Leger, 1999) présentées à la prochaine section, selon les quatre catégories du référentiel, permettant ainsi de tenir compte des orientations de la formation des enseignants et des changements en cours dans le milieu scolaire. 


\subsection{Catégorie 1 : Fondements}

La catégorie liée aux fondements des CP fait référence à l'enseignant en tant que professionnel, héritier d'une culture propre à ses fonctions, critique et interprète d'objets de savoir à enseigner (CP 1 et CP 2). Dans cette perspective, l'analyse des besoins de FC chez les enseignants d'ÉPS relève particulièrement de l'ajout du volet santé au programme d'ÉPS. Malgré ces nouveaux savoirs intégrés à l'ÉPS, plusieurs enseignants et chercheurs ont argumenté que l'éducation physique agit implicitement sur la santé de l'élève à l'école (Nutbeam, 1998; St Leger, 1999) et diverses études s'y sont intéressées. L'étude de Beaudoin et al. (2008) met en évidence la place de la santé dans les curricula scolaires québécois, ontariens et français. La santé transcende l'ensemble des programmes d'études et les disciplines offertes aux jeunes Canadiens et Français, mais il apparaît que l'ÉPS y joue un rôle de premier plan. L'analyse détaillée des curricula primaires des provinces canadiennes démontre de grandes disparités sur les aspects traitant des finalités de l'ÉS dans les programmes d'ÉPS et des contenus de l'ÉS abordés en ÉPS (Turcotte, Desbiens, Trudel, Demers et Roy, 2011). Ces auteurs concluent au rôle ambigu dévolu à l'ÉS, variable, voire instable, selon les provinces et questionnent les commandes ministérielles à propos de l'apport possible de l'ÉPS dans le domaine de la santé.

Par ailleurs, les études menées sur l'inclusion de l'ÉS en ÉPS montrent une multitude d'obstacles qui, aux yeux des enseignants d'ÉPS, influencent négativement son inclusion (Cogérino, 1999; Michaud, 2002; Turcotte et al., 2007). L'étude menée par Turcotte et al. (2007) démontre que parmi l'ensemble des savoirs essentiels intégrés au programme d'ÉPS, l'enseignant en privilégie seulement quelques-uns dont la pratique sécuritaire d'activités physiques, la condition physique et les structures et le fonctionnement du corps humain. Selon Cogérino, Marzin et Méchin (1998), peu d'enseignants d'ÉPS français ont procédé à un réaménagement des contenus puisque ces derniers reposent encore principalement sur la santé physique (Jourdan, Fiard, Mérini et Bertin, 2004). Un tel constat est-il lié à une tradition très marquée de l'ÉPS qui touche principalement la santé physique et qui aborde aujourd'hui de façon très timide la santé de façon holistique? C'est en quête d'éléments de réponses à ces questions que cette étude traite des besoins de FC en ÉS, tels qu'exprimés par des enseignants d'ÉPS.

\subsection{Catégorie 2: Acte d'enseigner}

Les quatre $\mathrm{CP}$ de cette catégorie ( $\mathrm{CP} 3$ à $\mathrm{CP}$ 6) mettent en évidence la complexité de l'acte d'enseigner et les diverses interactions avec les élèves. L'enseignant planifie et enseigne des contenus, supervise et organise les activités et les élèves et évalue les apprentissages de l'élève dans un climat propice à son développement. Tout comme le souligne Graber (2001), les compétences déployées au cœur des interactions amènent les enseignants à réviser leur rapport aux savoirs et aux compétences à développer chez l'élève. Toutefois, il semble que le renouvellement des pratiques professionnelles arrimées à une approche par compétences s'opère lentement (Lafortune et Lepage, 2007; Rioux-Dolan, 2004). Fullan (2003) précise que l'implantation de nouveaux programmes ne garantit pas à elle seule les changements inhérents à sa mise en œuvre telles qu'en témoignent des études en ÉPS (Fink et Stoll, 1998; Hargreaves, 2005). Turcotte (2006) propose que des éléments centraux, telles les conceptions et croyances des enseignants d'ÉPS et des éléments périphériques, tels les outils pédagogiques, influencent les pratiques pédagogiques. La question de l'évaluation attire aussi l'attention dans la littérature. Les travaux de Turcotte, Roy, Desbiens, Spallanzani, 
Goyette, Demers et Trudel (2008) n'ont pas permis d'observer de réelles évaluations reliées aux compétences dans les pratiques des enseignants d'ÉPS. Tout comme Rivard et Beaudoin (2005), Grenier, Boudreau, Raîche, Richard et Boudreault (2008) ont soulevé la nécessité de laisser du temps aux enseignants d'ÉPS pour modifier leurs pratiques évaluatives. Les travaux de Rivard, Porlier et Collet (2009) indiquent que l'utilisation des échelles de niveaux de compétences et la gestion des outils d'évaluation constituent les plus grandes difficultés relatives à l'acte d'évaluer à l'école secondaire.

Le décalage entre les pratiques pédagogiques des enseignants et les orientations ministérielles est largement documenté et relève d'une tradition behavioriste axée sur une formation compartimentée par objectifs fortement ancrée en enseignement (Brunelle, 2002), d'une nécessaire période d'appropriation du plan conceptuel (Hargreaves, 2005; Lafortune et Lepage, 2007) et des objets d'apprentissage (Turcotte et al., 2007), d'une forte résistance aux changements (Fullan, 2003) ou même d'un manque d'engagement dans les activités de FC (Armour et Yelling, 2007; Carlier et Mikulovic, 2003; Conseil supérieur de l'Éducation (CSÉ), 2007; Jonnaert, 2002). Peu importe le point de vue, les CP liées à l'acte d'enseigner se sont complexifiées et de nouveaux efforts doivent être déployés pour les arrimer dans l'esprit du renouveau pédagogique.

\subsection{Catégorie 3 : Contexte scolaire et social}

Un consensus émerge de la littérature: la dernière décennie a été marquée par une prise de conscience croissante de l'importance du rôle de l'environnement scolaire pour répondre aux besoins des jeunes. Cette troisième catégorie couvre un éventail de $\mathrm{CP}$ qui font écho à la nouvelle école québécoise: l'intégration des élèves à besoins particuliers en classes régulières ( $\mathrm{CP} 7)$, l'avancée des innovations technologiques (CP 8) et une collaboration accrue entre les partenaires concernés par la formation des jeunes (CP 9 et CP 10). Le référentiel propose donc quatre compétences reliées aux trois environnements qui composent la vie des jeunes, soit scolaire, familial et communautaire: «L'école n'a plus le monopole de la transmission et de la diffusion des savoirs, comme c'était le cas autrefois » (MEQ, 2001b, p.7). De nouveaux acteurs sont interpellés pour établir un partenariat devenu nécessaire avec les milieux scolaires dans une vision élargie de l'éducation (MEQ, 2001a); les enseignants sont ainsi amenés à partager leur responsabilité éducative. Des travaux menés en milieu scolaire révèlent que le partenariat ne va pas nécessairement de soi (Deslandes, 2001), mais la communication entre parents, enseignants et autres membres de l'école pourrait être la seule avenue possible pour favoriser le développement des jeunes, en l'occurrence leurs comportements de santé.

\subsection{Catégorie 4 : Identité professionnelle}

$\mathrm{Au}$ cours des dernières années, la société québécoise a traversé une série de changements modifiant le rôle des enseignants et nécessitant l'appropriation de $\mathrm{CP}$ de haut niveau qui ne peuvent être acquises au gré des essais et des erreurs (MEQ, 2001b). La complexité des situations et la mouvance des contextes éducatifs obligent l'enseignant à développer une expertise et à la maintenir dans le temps en prenant en charge sa FC. Ainsi, deux CP du référentiel sont reliées à cette quatrième catégorie et traitent de l'expertise et du professionnalisme attendus en enseignement (CP 11 et CP 12). 
Une tendance internationale met en évidence la variable optionnelle associée à la FC qui s'effectue sur une base volontaire malgré la diversité des contextes sociopolitiques, des dispositifs et des contenus. En ÉS, Aubry (2004) souligne l'importance du partenariat dans les formations dites intégrées à l'action et Sandrin-Berthon (2004) précise que ces formations devraient s'adresser à plusieurs catégories de personnes : enseignants, professionnels de la santé et travailleurs sociaux. Pour leur part, les enseignants d'ÉPS bénéficieraient davantage de contextes informels pour parfaire leur expertise afin d'échanger sur les expériences vécues avec leurs collègues et d'être accompagnés individuellement (Coppé et Schoonbroodt, 1992 ; Manidi et Dafflon-Arvanitou, 2000 ; Michaud, 2002). Puisque ces formations devraient être élaborées à partir des besoins exprimés par le personnel scolaire (Beaudoin et al., 2008; CSÉ, 2007; Lafortune et Lepage, 2007), une analyse approfondie des besoins des enseignants d'ÉPS nous paraît nécessaire pour actualiser la FC.

\section{Méthodologie}

La présente étude, qui a reçu l'approbation du comité d'éthique de l'Université de Sherbrooke, s'appuie sur une approche qualitative/interprétative afin de caractériser les besoins de FC des enseignants d'ÉPS au regard du référentiel des CP.

\subsection{Le recrutement des participants}

L'ensemble des écoles primaires du Québec $(n=1700)$ a reçu, entre avril et juin 2007, une enveloppe adressée à l'enseignant d'ÉPS et contenant un questionnaire, la lettre d'accompagnement présentant l'étude, le formulaire de consentement et une enveloppe de retour affranchie. Quatre semaines plus tard, une carte postale fut envoyée à l'ensemble des écoles en guise de rappel. De plus, les membres de la Fédération des éducateurs et éducatrices physiques enseignants du Québec ont été sollicités individuellement par courriel à deux reprises.

\subsection{Les participants}

Au total, 223 enseignants d'ÉPS de l'ordre d'enseignement primaire provenant de 54 des 62 commissions scolaires francophones ont répondu au questionnaire. Comparée aux statistiques du ministère de l'Éducation, du Loisir et du Sport pour l'année 2006-2007 (2008), la répartition hommes/femmes des participants à l'étude (64,1\% d'hommes et 35,9\% de femmes) est similaire à celle de la population des enseignants d'ÉPS québécois à l'ordre d'enseignement primaire (63,5\% d'hommes et 36,5\% de femmes). Par ailleurs, l'échantillon se compose d'enseignants d'ÉPS avec expériences diversifiées $(52,5 \%$ de 15 ans ou plus; $26,0 \%$ de six à 14 années et 21,5\% de cinq ans et moins).

\subsection{L'outil: le questionnaire}

Le questionnaire autoadministré développé et validé par Turcotte (2006) a été utilisé pour cette étude; le temps de passation était d'environ 60 minutes. Ce questionnaire a été examiné selon une méthode par jugements en vue de procéder à sa validation (Ghiglione et Matalon, 1998) en 
deux étapes. Dans un premier temps, la validation du contenu (objets traités et formulation des questions) a été réalisée par trois experts universitaires en éducation. Plusieurs modifications du questionnaire ont été apportées pour tenir compte des recommandations émises par ces experts. Dans un deuxième temps, quatre intervenants du domaine de l'éducation physique ont répondu au questionnaire individuellement. Par la suite, une rencontre individuelle avec chacun de ces intervenants a eu lieu pour obtenir leurs commentaires sur le contenu du questionnaire et sur la compréhension des questions. Quelques modifications ont été apportées au questionnaire avant de procéder à la collecte des données. Ces modifications touchaient principalement une meilleure précision des termes utilisés dans le programme de formation du ministère de l'Éducation afin de les rendre plus explicites et l'ajout de certains choix de réponses.

Précédé d'une introduction présentant les directives, le questionnaire comporte des questions offrant trois types de réponses: des échelles de réponses, des listes d'items à sélectionner ou des réponses à court développement. Cet article présente les réponses à deux de ces questions qui proviennent de la section traitant des impacts de leur enseignement en éducation à la santé.

\subsection{L’analyse des données}

Les propos des participants ont fait l'objet d'une analyse déductive (Landry, 1998) où les catégories prédéterminées correspondent aux 12 CP (MEQ, 2001b). La CP abordant la langue d'enseignement soit Communiquer clairement et correctement dans la langue d'enseignement, à l'oral et à l'écrit, dans les divers contextes liés à la profession enseignante ne figure pas dans les résultats puisqu'aucun énoncé n'y était relié.

Dans un premier temps, les 280 énoncés provenant de 223 questionnaires ont été classés, de façon indépendante, par deux membres de l'équipe de recherche (taux d'accord de 87,8\%). Les 34 énoncés classés différemment ont été reclassés par consensus, permettant d'ajuster les définitions des catégories. Dans un deuxième temps, deux autres personnes ont classé, de façon indépendante et à l'aveugle, un échantillon de 53 énoncés dont la répartition dans les catégories était proportionnelle au nombre d'énoncés en lien avec chacune des $12 \mathrm{CP}$. Ces codeurs ont obtenu un taux d'accord de $88,7 \%$ et $81,1 \%$.

\section{Résultats}

La nécessité d'offrir des FC en lien avec la compétence disciplinaire Adopter un mode de vie sain et actif préoccupe la majorité des enseignants d'ÉPS de notre enquête. La presque totalité $(93,7 \%)$ des 223 participants, i.e. 205, ont répondu par l'affirmative quant à l'importance d'offrir des FC aux enseignants d'ÉPS. Au total, 163 d'entre eux ont émis des suggestions (280 énoncés) quant aux éléments de contenu des FC (tableau 1). Leurs besoins de FC tel que répartis en fonction du référentiel indiquent une prédominance de la catégorie Acte d'enseigner (69,3\%) comparativement aux trois autres catégories de compétences professionnelles à savoir Fondements $(15,7 \%)$, Contexte social et scolaire $(8,6 \%)$ et Identité professionnelle $(6,4 \%)$. 
Tableau 1

Répartition des besoins de FC selon les compétences professionnelles (MEQ, 2001b)

Catégories de compétences professionnelles (provenance des énoncés)

Fréquence

№ Compétences professionnelles

\section{Fondements (44 enseignants, 44 énoncés)}

CP 1 - Agir en tant que professionnel ou professionnel héritier, critique et interprète d'objets de savoir ou de culture dans l'exercice de ses fonctions

CP 2 - Communiquer clairement et correctement dans la langue d'enseignement, à l'oral et à l'écrit, dans les divers contextes liés à la profession enseignante

\section{Acte d'enseigner (136 enseignants, 194 énoncés)}

CP 3 - Concevoir des situations d'enseignement pour les contenus à faire apprendre, et ce, en fonction des élèves concernés et du développement des compétences visées dans le programme de formation

CP 4 - Piloter des situations d'enseignement pour les contenus à faire apprendre, et ce, en fonction des élèves concernés et du développement des compétences visées dans le programme de formation

CP 5 - Évaluer la progression des apprentissages et le degré d'acquisition des compétences des élèves pour les contenus à faire apprendre

CP 6 - Planifier, organiser et superviser le mode de fonctionnement du groupe-classe en vue de favoriser l'apprentissage et la socialisation des élèves

\section{Contexte social et scolaire (22 enseignants, 24 énoncés)}

CP 7 - Adapter ses interventions aux besoins et aux caractéristiques des élèves présentant des difficultés d'apprentissage, d'adaptation ou un handicap

CP 8 - Intégrer les technologies de l'information et des communications aux fins de préparation et de pilotage d'activités d'enseignement-apprentissage, de gestion de l'enseignement et de développement professionnel

CP 9 - Coopérer avec l'équipe-école, les parents, les différents partenaires sociaux et les élèves en vue de l'atteinte des objectifs éducatifs de l'école

CP 10 - Travailler de concert avec les membres de l'équipe pédagogique à la réalisation des tâches permettant le développement et l'évaluation des compétences visées dans le programme de formation, et ce, en fonction des élèves concernés

\section{Identité professionnelle (18 enseignants, 18 énoncés)}

CP 11 - S'engager dans une démarche individuelle et collective de développement professionnel

CP 12 - Agir de façon éthique et responsable dans l'exercice de ses fonctions

$3(1,1 \%)$ 


\subsection{Catégorie 1 : Fondements}

Les énoncés recueillis pour cette catégorie de compétence sont uniquement en lien avec l'agir professionnel (CP 1). D'une part, plusieurs enseignants d'ÉPS s'interrogent sur le contenu à enseigner en lien avec les savoirs essentiels présents dans les programmes de formation : «Identifier les exigences minimales en matière de condition physique, d'alimentation, de sommeil, etc.»; "Quelques idées sur les thèmes à toucher avec les élèves: hygiène, premiers soins, alimentation et gestion du stress ». D'autre part, certains se questionnent quant à la répartition des contenus selon les cycles: "Une meilleure structuration serait la bienvenue avec ce qu'il faut vraiment voir pour chacun des cycles »; qu'ils relient parfois à l'identification des objets d'apprentissage: "Quelle connaissance doit-on transmettre et à quel niveau?». Par ailleurs, les composantes des compétences disciplinaires enseignées à l'élève et leurs interrelations nécessitent certaines clarifications. Enfin, la compréhension générale du domaine de l'ÉS les préoccupe également: «Des études valides en lien avec cette compétence et son influence sur les jeunes»; "Avoir de la documentation récente».

\subsection{Catégorie 2: Acte d'enseigner}

La CP 3 correspond à la conception de situations d'enseignement et elle est celle qui regroupe le plus d'énoncés soulevant deux besoins prioritaires pour les enseignants d'ÉPS : a) l'élaboration de nouvelles situations d'enseignement ou l'utilisation de situations déjà élaborées favorisant la progression des apprentissages; et b) la disponibilité d'outils pédagogiques pour supporter la mise en œuvre des situations d'enseignement. L'élaboration de situations d'enseignement peut correspondre au besoin d'acquérir de nouvelles façons d'élaborer ces dernières: "La formation devrait reposer sur la façon de créer des activités thématiques en lien avec les divers projets du volet santé qui sont proposés durant l'année». Il est également question du partage de situations d'enseignement créées par d'autres collègues: "Des exemples de situations d'enseignement déjà vécues dans un gymnase». Enfin, la conception de situations d'enseignement est reliée à la possibilité d'adapter les situations aux besoins de l'élève: «Des mises en situation signifiantes pour chaque cycle»; ou aux différents cycles d'études afin d'assurer la progression des apprentissages: «J'aimerais qu'il existe de la FC au $1^{\text {er }}$ cycle du primaire. Souvent, les formations auxquelles j'ai assisté étaient pour des clientèles des $2^{\mathrm{e}}$ et $3^{\mathrm{e}}$ cycles du primaire».

La disponibilité d'outils pédagogiques signifie l'accès à une variété d'outils supportant le déploiement des situations d'enseignement en considérant les savoirs essentiels en ÉS et les caractéristiques de l'élève. Certains facteurs contextuels influencent les besoins exprimés tels que le temps: "Des outils à remplir par les élèves nécessitant peu de temps à compléter par eux»; l'uniformisation: "Un document généralisé à toute la commission scolaire et adaptable au besoin»; l'environnement d'enseignement: «Des outils concrets de planification en lien avec les pratiques des jeunes à l'extérieur de l'école»; et un désir d'autogestion de l'élève: «Une variété d'outils pour stimuler le jeune à prendre soin de sa santé». Enfin, est évoqué le besoin de suivre des formations à propos des modalités d'utilisation et de suivi d'outils pédagogiques comme le portfolio et le carnet santé. 
Trois besoins de formation ont été identifiés parmi les énoncés recueillis en lien avec le pilotage des situations d'enseignement (CP 4). D'abord, les participants s'interrogent sur la pertinence des capsules santé, les moyens à privilégier dans l'intervention pédagogique en ÉS et les modalités à privilégier pour que le jeune poursuive son apprentissage à la maison: «Ma formation est très récente et je me pose encore souvent des questions à savoir comment bien intégrer les concepts santé dans mon gymnase. Je vois encore beaucoup de collègues qui modifient des jeux pour se donner bonne conscience ou par manque de formation afin d'intégrer la santé. Je ne suis pas convaincu que de jouer à la «carotte et au céleri» à la place «du policier et des voleurs» ait un impact important sur les jeunes». Le deuxième besoin exprimé par les participants fait écho aux aspects théoriques de l'ÉS et à leurs impacts sur le temps actif de l'élève lors des cours d'ÉPS : «L'effet de l'instauration de la théorie dans le cours versus mettre les élèves en action. Est-ce qu'on leur enlève du temps d'action?». Troisièmement, les enseignants souhaitent l'identification de stratégies facilitant l'acquisition d'habitudes de vie: «Il faut démystifier l'ampleur de la tâche et cerner les interventions les plus efficaces dans la poursuite d'acquisition de saines habitudes de vie».

L'identification de moyens pertinents de suivi de la progression de l'élève et d'évaluation des attentes de fin de cycle constitue deux éléments nécessitant des éclaircissements lorsqu'il est question d'évaluation des élèves (CP 5). Des enseignants d'ÉPS se questionnent sur les moyens assurant un suivi judicieux des progrès de l'élève: «Cahier pour suivre l'évolution de l'enfant». D'autres souhaitent des moyens concrets pour évaluer cette compétence à l'extérieur de l'école : «Comment pouvoir poser un jugement juste et précis à Adopter un mode de vie sain et actif? [...] que pouvonsnous mettre en place pour assurer des modifications de comportements à la maison, sur le sommeil et la nutrition?». Enfin, ils demandent du matériel pédagogique pour l'évaluation, une formation sur la conception des outils : «Elles devraient aussi nous permettre d'être en mesure de concevoir des outils d'évaluation pertinents et réalisables dans ce domaine»; et sur l'établissement d'un bilan des acquis leur permettant de porter un jugement sur le degré d'acquisition des compétences: «Recevoir des documents simples pour réaliser le bilan des élèves ».

Dans une moindre importance, les besoins de formation sont en lien avec le mode de fonctionnement du groupe-classe (CP 6) et reposent sur des activités usuelles de la classe, telles que: 1) la gestion du temps de classe en ÉS; 2) la gestion des carnets santé; et 3) la gestion des élèves en situation de co-enseignement. La dimension temporelle est donc une constante pour l'ensemble des énoncés de cette catégorie.

\subsection{Catégorie 3 : Contexte social et scolaire}

Pour adapter leurs interventions aux besoins et aux caractéristiques de l'élève (CP 7), les enseignants d'ÉPS mentionnent la nécessité de participer à des formations pour développer des situations d'enseignement et du matériel pédagogique utiles dans différents milieux socioéconomiques et adaptés à diverses clientèles. À propos de la compétence liée aux technologies de l'information et des communications (CP 8), les enseignants d'ÉPS se demandent comment ces technologies pourraient soutenir l'ÉS en milieux scolaire et familial. En ce qui concerne les besoins de formation reliés à la compétence qui traite du partenariat (CP 9), les enseignants d'ÉPS se questionnent à la fois sur l'implication des parents : «Comment impliquer la famille? 
Bien souvent, les jeunes veulent s'impliquer, mais les parents ne les suivent pas »; et des autres partenaires présents à l'école notamment la direction, les infirmières, les spécialistes et les enseignants titulaires: "Pouvoir se rencontrer entre professeurs du même milieu scolaire pour échanger sur les aspects et les thématiques développés ainsi que sur les moyens efficaces».

Enfin, travailler de concert avec les membres de l'équipe pédagogique (CP 10) suppose que l'ensemble des intervenants s'implique en ÉS. Or, les besoins exprimés par certains participants révèlent la nécessité de proposer des FC à l'ensemble de l'équipe-école afin qu'un plus grand nombre d'intervenants puissent contribuer substantiellement à l'ÉS. Ces enseignants d'ÉPS se questionnent sur la nature interdisciplinaire de la compétence disciplinaire Adopter un mode de vie sain et actif et sur la contribution des autres membres de l'équipe pédagogique à sa mise en œuvre dans l'environnement scolaire.

\subsection{Catégorie 4 : Identité professionnelle}

Pour s'engager dans une démarche de développement professionnel (CP 11), les enseignants d'ÉPS soulignent que les activités de FC permettent d'échanger et de réfléchir sur les choix pédagogiques opérés en ÉS. Ils souhaitent partager leurs idées pédagogiques, leurs expériences vécues: «Permettre de nous rencontrer entre éducateurs physiques pour favoriser les échanges»; et élaborer collectivement des projets pédagogiques: «Je voudrais travailler avec des groupes d'enseignants afin de construire et de discuter sur la compétence Adopter un mode de vie sain et actif ». Enfin, certaines conditions semblent nécessaires afin de faciliter leur engagement dans une démarche de développement professionnel. Les enseignants ont besoin qu'une variété de formations dans l'ensemble des commissions scolaires soit offerte, que des ressources financières soient accordées à la suppléance lors des formations et que le temps dévolu à la formation soit suffisant pour engendrer un réinvestissement dans leur pratique professionnelle.

La compétence qui renvoie à l'agir de manière éthique et responsable dans l'exercice de ses fonctions (CP 12) est grandement influencée par les normes communes partagées par un groupe de professionnels. Or, il ne fait aucun doute que l'inclusion de l'ÉS en ÉPS modifie les normes communes partagées par les enseignants d'ÉPS et conduit à un questionnement à l'égard des pratiques professionnelles dont la contribution attendue de l'ÉPS à l'amélioration de l'état de santé des jeunes. La réflexion suivante illustre bien cette préoccupation: "Le développement autonome de la profession d'éducateur physique face à l'ÉS sans obligation ou dépendance face aux organisations politiques ou aux associations sportives qui créent des événements à la mode». De plus, lorsque l'ÉS interfère avec les comportements et les valeurs véhiculés par la famille, les enseignants d'ÉPS se demandent comment assurer le respect de l'élève en toute équité: «On doit mieux cerner la problématique entourant cette compétence et le rôle des parents. On a souvent l'impression d'évaluer la famille et non l'élève». Enfin, éduquer à la santé implique l'engagement personnel et la cohérence entre les actions professionnelles dans et hors école. Réfléchir collectivement sur les valeurs à partager est un besoin de formation exprimé: «Je crois que si l'on veut enseigner aux élèves à Adopter un mode de vie sain et actif, il faut nous-mêmes en être les précurseurs». 


\section{Discussion}

D'entrée de jeu, les enseignants d'ÉPS interrogés sont unanimes quant à l'importance d'offrir aux spécialistes des FC en lien avec la compétence disciplinaire Adopter un mode de vie sain et actif. Les besoins et les contenus attendus de ces formations semblent être associés, en proportion variable, à chacune des catégories du référentiel.

\subsection{Catégorie 1 : Fondements}

Les résultats de l'étude permettent de corroborer plusieurs conclusions de travaux scientifiques menés antérieurement et de mieux comprendre les besoins que la $\mathrm{FC}$ aura à considérer dans un avenir rapproché. Les enseignants qui ont pris part à notre étude formulent de nombreuses questions à l'égard de l'ÉS dans le curriculum scolaire de l'ÉPS. À leur façon, ils s'expriment sous l'angle des fondements. Qu'est-ce que l'ÉS ? Quels sont les savoirs essentiels à enseigner et leurs contenus respectifs? Tel que discuté par Turcotte et al. (2011), le rôle ambigu dévolu à l'ÉS en ÉPS peut expliquer le questionnement des enseignants d'ÉPS à ce sujet. Ainsi, l'expression de leurs besoins soulève l'urgence d'énoncer une conception de l'ÉS, sa contribution quant aux savoirs à enseigner et ses contenus à structurer par cycle d'enseignement.

À notre avis, il importe de mieux définir l'ÉS en tant que champ d'études par une vaste réflexion collective impliquant la communauté scientifique et les praticiens puisque de nombreuses études ont dénoncé l'approche restreinte des enseignants d'ÉPS entourant l'ÉS, limitée souvent à la pratique sécuritaire d'activités physiques et sportives ou à la condition physique (Beaudoin et al., 2008; Cogérino, 1999; Jourdan et al., 2004).

\subsection{Catégorie 2: Acte d'enseigner}

Les participants ont aussi abordé la nécessité d'acquérir des outils pédagogiques pour faciliter la mise en œuvre de situations d'enseignement dans une approche par compétences. Bien que cette quête d'outils intimement liée à la conception semble légitime chez les praticiens, certains chercheurs suggèrent qu'elle doit plutôt faire suite à une période d'appropriation du renouveau pédagogique (CSÉ, 2007; Lafortune et Lepage, 2007; Rivard et al., 2009), puisqu'il serait prématuré de fournir aux enseignants en exercice des outils pédagogiques de type clé en main si les fondements épistémologiques qui sous-tendent ce renouveau demeurent dans l'ambiguïté. Par ailleurs, Michaud (2002) précise que les besoins exprimés par les enseignants d'ÉPS concernent non seulement des activités de formation proprement dites, mais aussi un accompagnement à l'élaboration de matériels didactiques et au suivi quant à l'évolution de projets scolaires. Il nous apparaît qu'un tel constat offre une piste à privilégier pour assurer une période d'appropriation assistée du renouveau pédagogique. Le modèle de changement chez l'enseignant de Guskey $(2002,2003)$ et les travaux de Fiszer (2004), qui s'intéressent au développement professionnel de la population enseignante, font également état de ce besoin de suivi continu dans le but de supporter ou même d'inciter les efforts nécessaires à tout changement qu'impliquent les réformes scolaires. Le support apparaît nécessaire pour tolérer l'anxiété d'échecs occasionnels, indissociables de toute démarche d'exploration de nouvelles approches pédagogiques et l'incitation pour contrer la résistance, encourager, motiver et appuyer la persévérance face aux défis qui se présentent (Guskey, 2002). 
Les enseignants soulèvent aussi les enjeux de l'évaluation, en particulier les attentes de fin de cycle et la difficulté d'évaluer l'adoption d'un mode de vie sain et actif à l'extérieur de l'environnement scolaire. Ces résultats corroborent ceux issus de travaux menés antérieurement (Grenier et al., 2008; Rivard et al., 2009; Turcotte et al., 2008).

\subsection{Catégorie 3: Contexte social et scolaire}

Les enseignants interrogés sont également interpellés par le rôle et l'implication de divers acteurs en ÉS. Ces derniers mentionnent rechercher des moyens pour inciter les parents à collaborer aux projets éducatifs de l'école; pour multiplier les moments d'échange avec d'autres partenaires scolaires tels les enseignants titulaires ou autres spécialistes et d'autres collègues de leur discipline. L'idée d'un nouveau partenariat soulevée ici est de plus en plus présente dans la littérature et semble devenir indispensable pour toute initiative scolaire en ÉS (Bizzoni-Prévieux, 2011; Mérini, 2004). S'inscrivant également dans la mission du renouveau pédagogique, la mise en place d'une action concertée apparaît comme une stratégie à privilégier afin d'aider les enseignants à travailler davantage en collaboration les uns avec les autres.

Toutefois, l'établissement d'un tel partenariat pose plusieurs défis. Par exemple, les enseignants soulèvent la problématique d'avoir à éduquer la famille autant que l'élève. Dans le contexte de l'étude, les enseignants expriment plus spécifiquement le besoin de savoir comment impliquer les parents dans l'acquisition de la compétence disciplinaire Adopter un mode de vie sain et actif; et particulièrement lorsque le potentiel de l'élève est limité au sein de son environnement familial. Ces résultats rejoignent ceux de Deslandes (2001) qui énonce les défis à la création d'un partenariat positif entre l'école et la famille.

Puis, en lien avec d'autres partenaires scolaires et collègues de leur discipline, les enseignants souhaitent davantage coordonner leurs actions dans la mise en place de dispositifs reliés à la santé et échanger sur leurs expériences vécues. Les travaux d'Armour et Yelling (2007) et de Fiszer (2004) soulignent l'importance de développer une communauté professionnelle d'apprentissage pour offrir une multitude d'occasions d'assurer des apprentissages informels dans un contexte de collaboration entre collègues. Toutefois, très peu d'études se sont particulièrement arrêtées aux dispositions à mettre en place pour permettre d'instaurer cette collaboration souhaitée dans le contexte de l'ÉPS pour l'ÉS. Deux études conduites auprès d'enseignants d'ÉPS en Angleterre (Duncombe et Armour, 2004) et au Canada (Beaudoin et al., 2006) illustrent plusieurs conditions pouvant nuire à la mise en place d'une communauté professionnelle d'apprentissage, dont les priorités, les structures et les procédures du milieu scolaire. Il nous apparaît donc qu'une nouvelle culture de collégialité est à développer dans le réseau scolaire québécois et le renouveau pédagogique offre un prétexte fertile en matière de projets réunissant plusieurs acteurs.

\subsection{Catégorie 4 : Identité professionnelle}

Et si la période d'appropriation du renouveau pédagogique, tant dénoncée dans la communauté scientifique, était mise au service de la construction de l'identité professionnelle des enseignants? De fait, les valeurs à véhiculer et le rôle de modèle que l'enseignant est amené à jouer dans le contexte d'une ÉS en milieu scolaire ne sont pas sans interpeller plusieurs des participants de cette 
étude. Si réfléchir collectivement sur les valeurs à partager est un besoin de formation exprimé, des chercheurs comme Tinning (2004) et Macdonald, Hunter et Carlson (2002) soulèvent le rôle des croyances et des valeurs des enseignants dans la construction de leur identité professionnelle. Dans le contexte de la formation initiale à l'ÉS en ÉPS, Tinning (2004) discute de la nécessité d'une nouvelle façon de voir et de penser la santé afin de pouvoir construire une nouvelle identité à la fois personnelle et professionnelle. À ce propos, notre étude s'inspire d'un postulat associé à l'éducation à la citoyenneté: «on enseigne ce que l'on est», emprunté à Perrenoud et cité par le CSE (1998, p. 75). Une telle vision prend toute son importance dans l'enseignement de l'ÉPS, car elle soulève les défis que présente l'enseignement de la compétence disciplinaire Adopter un mode de vie sain et actif, touchant l'enseignant dans son propre mode de vie, à l'intérieur comme à l'extérieur de l'école. Ce défi d'enseignant modèle en ÉPS, qui concerne une sphère très intime de la personne, a d'ailleurs été soulevé par Rivard et Trudeau (2006) dans le cadre de leur étude portant sur l'engagement d'étudiants en enseignement de l'ÉPS dans la réalisation d'une démarche expérientielle axée sur cette compétence disciplinaire. Leurs résultats révèlent qu'une stratégie de formation impliquant une démarche expérientielle a l'avantage de sensibiliser les futurs enseignants aux éventuelles situations en enseignement de l'ÉPS où ils auront à leur tour à stimuler la motivation des élèves qui devront s'engager dans une démarche santé. Il nous apparaît donc qu'une telle démarche expérientielle est à explorer en FC.

\section{Conclusion}

Les besoins de FC en matière d'ÉS pour les enseignants d'ÉPS sont nombreux et variés. Rappelons que la conception d'outils pédagogiques et l'évaluation constituent leurs plus grands besoins en FC, mais qu'ils souhaitent également une formation qui assurerait un accompagnement individualisé, tout en créant un nouveau partenariat avec d'autres collègues. Les résultats de cette étude permettent d'aller au-delà du décalage entre les pratiques pédagogiques et les orientations ministérielles et d'identifier certains paramètres qui sous-tendent cette réalité. Les témoignages des enseignants d'ÉPS s'inscrivent dans une perspective socioconstructiviste, car ils souhaitent favoriser, sur une base collective, l'élaboration de nouvelles situations d'enseignement, adaptables aux besoins de l'élève, transférables à divers contextes de pratique et favorisant l'autogestion personnelle. À cet égard, il s'avérerait pertinent de différencier les principaux besoins de FC d'enseignants d'ÉPS en fonction de certaines variables comme les caractéristiques du milieu scolaire, les expériences d'enseignement et le sexe.

Ainsi, il nous apparaît juste de conclure que la FC telle qu'offerte présentement aux enseignants d'ÉPS n'a pas su entièrement répondre à leurs besoins. La méta-analyse de Guskey (2003) a permis d'identifier 21 caractéristiques à considérer pour l'établissement d'un modèle de développement professionnel pour les enseignants, dont l'importance d'améliorer les connaissances pédagogiques et les répertoires des contenus à enseigner, de fournir suffisamment de temps et de ressources et de promouvoir la collégialité et la collaboration. Fiszer (2004) souligne qu'un modèle de développement professionnel continu nécessite une très grande collaboration qui implique un énorme changement de culture qui doit se faire à l'intérieur des milieux scolaires. Il nous apparaît que ce changement de culture pourrait débuter par la prise en compte des besoins exprimés par les enseignants d'ÉPS. Plusieurs pistes d'action à privilégier ont été énoncées afin d'amener les praticiens à coconstruire des connaissances ancrées dans leurs pratiques pédagogiques, tout en 
clarifiant les valeurs et les convictions personnelles qui portent leurs actions. Dans cet esprit, la recherche-action ou la recherche collaborative amèneraient les praticiens à prendre place au cœur de la recherche à titre de cochercheurs, là où leur double rôle leur permettrait, à la fois, d'apporter un regard de l'intérieur et de poser un regard réflexif sur leur pratique.

\section{Références}

Armour, K. et Yelling, M. (2007). Effective professional development for physical education teachers: The role of informal, collaborative learning. Journal of Teaching in Physical Education, 26, 177-200.

Aubry, B. (2004). La formation des enseignants en IUFM: articulation formation initiale et formation continue. In D. Jourdan (dir.). La formation des acteurs de l'éducation à la santé en milieu scolaire (p. 205-221). Toulouse: Éditions universitaires du Sud.

Beaudoin, C., Rivard, M.-C., Grenier, J. et Caty, D. (2008). Les enjeux de l'intégration de l'éducation à la santé à l'école primaire: l'apport des ÉPS au Québec, en Ontario et en France. In J.-F. Gréhaigne, P. Godbout, N. Wallian, M.-P. Poggi et M. Musard (dir.), Coconstruire des savoirs. Les métiers d'intervenants dans les APS (p. 317-333). Besançon: Presses universitaires de Franche-Comté.

Beaudoin, C., Trudel, P. et Mathias, M. (2006). L'implantation d'un nouveau programme-cadre en éducation physique et à la santé: l'expérience des enseignants en milieux francophones ontariens. La revue canadienne de l'éducation, 29(4), 923-948.

Bechtel, P. A. et O'Sullivan, M. (2007). Enhancers and inhibitors of teacher change among secondary physical educators. Journal of Teaching in Physical Education, 26(3), 221-235.

Bizzoni-Prévieux, C. (2011) Les partenariats en éducation à la santé à l'école primaire: analyse comparée entre le Québec et la France, Thèse de doctorat en éducation, Faculté des sciences de l'éducation, Université du Québec à Montréal.

Brunelle, J. (2002). L'intervention en activité physique et sportive: un point de vue sur la recherche nord-américaine, AVANTE, 8(2), 1-13.

Carlier, G. et Mikulovic, J. (2003). Activité physique et santé en milieu scolaire: le débat est ouvert. La santé de l'homme, 364, 23-24.

Cogérino, G. (1999). Apprendre à gérer sa vie physique. Paris: Presses universitaires de France.

Cogérino, G., Marzin, P. et Méchin, N. (1998). Pratiques et représentations chez les enseignants d'éducation physique et sportive et de sciences de la vie et de la terre. Recherche et Formation, 28, 9-28.

Conseil supérieur de l'Éducation (CSÉ). (1998). Éduquer à la citoyenneté. Rapport annuel 1997-1998 sur l'état et les besoins de l'éducation. Québec: Gouvernement du Québec.

Conseil supérieur de l'Éducation (CSÉ). (2007). Soutenir l'appropriation des compétences transversales et des domaines généraux de formation. Québec: Gouvernement du Québec.

Coppé, M. et Schoonbroodt, C. (1992). Guide pratique d'éducation pour la santé. Bruxelles: De Boeck Université.

Deslandes, R. (2001). A vision of home-school partnership: three complementary conceptual frameworks. In F. Smit, K. van der Wolf et P. Sleegers (dir.), A bridge to the future collaboration between parents, schools and communities. Parent participation (p. 11-24). Stichting Katholieke Universiteit te Nijmegen: ITS.

Duncombe, R. et Armour, K. M. (2004). Collaborative professional learning: from theory to practice. Journal of Inservice Education, 30(1), 141-166.

Fink, D. et Stoll, L. (1998). Educational change: Easier said than done. In A. Hargreaves, A. Leiberman, M. Fullan, et D. Hopkins (dir.), International Handbook of Educational Change (p. 297-321). Boston, MA: Kluwer Academic Publishers. 
Fiszer, E.-P. (2004). How teachers learn best? An Ongoing Professional Development Model. Lanham, MD : Scarecrow Education.

Fullan, M. (2003). Changes Forces: With a vengeance. New York, NY: Routledge Falmer Press.

Ghiglione, R. et Matalon, B. (1998). Les enquêtes sociologiques : théories et pratiques. Paris : Armand Colin.

Graber, K.-C. (2001). Reasearch on Teaching in Physical Education. In V. Richardson (dir.), Handbook of research on teaching, (4 éd.) (p. 491-519). Washington: Educational Research Association.

Grenier, J., Boudreau, P., Raîche, G., Richard, J.-F. et Boudreault, I. (2008). Notre évaluation est-elle authentique? Propulsion, 20(2), 8-10.

Guskey, T. R. (2002). Professional Development and Teacher Change. Teachers and Teaching: theory and practice 8(3/4), 381-391.

Guskey, T. R. (2003). The Characteristics of Effective Professional Development: A Synthesis of Lists. Communication présentée à l'Annual meeting of the American Educational Research Association, Chicago, IL, 21-25 avril.

Hargreaves, A. (2005). Educational change takes ages: Life, career and generational factors in teachers' emotional response to educational change. Teacher and Teaching Education, 21(8), 967-983.

Johns, D.-P. (2003). Changing the Hong Kong physical education curriculum: A post-structural case study. Journal of Educational Change, 4(4), 345-368.

Jonnaert, P. (2002). Compétences et socioconstructivisme. Un cadre théorique. Bruxelles: De Boek Université.

Jourdan, D. (2004). Introduction. In D. Jourdan (dir.), La formation des acteurs de l'éducation à la santé en milieu scolaire (p. 19-24). Toulouse : Éditions universitaires du Sud.

Jourdan, D., Fiard, J., Mérini, C. et Bertin, F. (2004). Practices and representations of secondary school physical education toward health education. Communication présentée au $9^{\mathrm{e}}$ Congrès annuel de l'ECSS, ClermontFerrand, 12 juillet.

Lafortune, L. et Lepage, C. (2007). Une expérience d'accompagnement socioconstructiviste d'un changement en éducation. Des orientations à réinvestir dans d'autres contextes. In L. Lafortune, M. Ettayebi et P. Jonnaert (dir.), Observer les réformes en éducation (p. 33-52). Québec: Presses de l'Université du Québec.

Landry, R. (1998). L'analyse de contenu. In B. Gauthier (dir.), Recherche sociale. De la problématique à la collecte de données (p. 329-356). Sainte-Foy: Presses de l’Université du Québec.

Macdonald, D., Hunter, L. et Carlson, T. (2002). Teacher knowledge and the disjunction between school curricula and teacher education. Asia-Pacific Journal of Teacher Education, 30(3), 259-275.

Manidi, M.-J. et Dafflon-Arvanitou, I. (2000). Activité physique et santé. Apports des sciences humaines et sociales. Éducation à la santé par l'activité physique. Paris: Masson.

Mérini, C. (2004). Former au partenariat, est-ce possible? In D. Jourdan (dir.), La formation des acteurs de l'éducation à la santé en milieu scolaire (p. 161-182). Toulouse : Éditions universitaires du Sud.

Michaud, V. (2002). L'intégration de l'éducation à la santé dans les programmes d'éducation physique du primaire et $d u$ secondaire, Thèse de doctorat en psychopédagogie, Faculté des sciences de l'éducation, Université Laval, Sainte-Foy.

Ministère de l'Éducation du Québec (MEQ) (1999). Orientations pour la formation continue du personnel enseignant. Choisir plutôt que subir le changement. Québec: Gouvernement du Québec.

Ministère de l'Éducation du Québec (MEQ) (2001a). Programme de formation de l'école québécoise. Éducation préscolaire, enseignement primaire. Québec: Gouvernement du Québec.

Ministère de l'Éducation du Québec (MEQ) (2001b). La formation à l'enseignement, les orientations et les compétences professionnelles. Québec: Gouvernement du Québec. 
Ministère de l'Éducation du Québec (MEQ) (2003). Programme de formation de l'école québécoise. Enseignement secondaire, premier cycle. Québec: Gouvernement du Québec.

Ministère de l'Éducation, du Loisir et du Sport (MELS) (2007). Programme de formation de l'école québécoise. Enseignement secondaire, deuxième cycle. Québec: Gouvernement du Québec.

Ministère de l'Éducation, du Loisir et du Sport (MELS) (2008). Statistiques de l'éducation. Enseignement primaire, secondaire, collégial et universitaire. Québec: Gouvernement du Québec.

Nutbeam, D. (1998). Evaluating health promotion: progress, problems and solutions. Health Promotion International, 13(1), 27-44.

Rioux-Dolan, M. (2004). L'accompagnement du personnel enseignant. Enjeux et défis dans le contexte de la réforme. In M. L'Hostie et L.-P. Boucher (dir.), L'accompagnement en éducation. Un soutien au renouvellement des pratiques (p. 13-30). Québec: Presses de l’Université du Québec.

Rivard, M.-C. et Beaudoin, C. (2005). Physical Education in Canada. In U. Puhse et M. Gerber (dir.), An international comparison of physical education: concepts, problems, prospects (p. 150-173). Meyer \& Meyer Sport.

Rivard, M.-C., Porlier, P. et Collet, M. (2009). Les pratiques évaluatives au 1er cycle du secondaire: tendances en éducation physique et à la santé. Rapport de recherche remis au Ministère de l'Éducation, du Loisir et du Sport.

Rivard, M.-C. et Trudeau, F. (2006). Description de l'engagement d'étudiants en enseignement de l'éducation physique et à la santé dans la réalisation d'un contrat axé sur la compétence disciplinaire santé. Res Academica, 24(2), 123137.

Sandrin-Berthon, B. (2004). Les formations en éducation à la santé en France: état des lieux et recommandations. In D. Jourdan (dir.), La formation des acteurs de l'éducation à la santé en milieu scolaire (p. 67-92). Toulouse: Éditions universitaires du Sud.

St Leger, L. (1999). The opportunities and effectiveness of the health promoting primary school in child health - a review of the claims and evidence. Health Education Research, 14(1), 51-69.

Tinning, R. (2004). Rethinking the preparation of HPE teachers: ruminations on knowledge, identity, and ways of thinking. Asia-Pacific Journal of Teacher Education, 32(3), 241-253.

Turcotte, S. (2006) L'inclusion de l'éducation à la santé en éducation physique: analyse des pratiques pédagogiques d'éducateurs physiques du primaire, Thèse de doctorat éducation, Faculté des sciences de l'éducation, Université du Québec à Montréal.

Turcotte, S., Desbiens, J.-F., Trudel, C., Demers. J. et Roy, M. (2011). L'inclusion de l'éducation à la santé dans les programmes canadiens d'éducation physique. Revue phénEPS, 3(1), 1-21.

Turcotte, S., Gaudreau, L. et Otis, J. (2007). Les objets d'enseignement-apprentissage: éléments d'illustration de l'inclusion de l'éducation à la santé en éducation physique. STAPS, 75, 115-129.

Turcotte, S., Roy, M., Desbiens, J.-F., Spallanzani, C., Goyette, R., Demers, J. et Trudel, C. (2008). Les pratiques d'évaluation de l'enseignement en éducation à la santé d'éducateurs physiques du primaire. Communication présentée à la $5^{\mathrm{e}}$ Biennale de l'Association pour la recherche sur l'intervention en sport (ARIS), Rodez, 14-16 mai. 